\title{
Applicability of Endobronchial Ultrasound and Virtual Bronchoscopic Navigation Combined with Rapid On-Site Evaluation in Diagnosing Peripheral Lung Lesions
}

Jia-Chao Qi

Zhangzhou Affiliated Hospital of Fujian Medical University

Liping Liao

Zhangzhou Affiliated Hospital of Fujian Medical University

Zhiwei Zhao

Zhangzhou Affiliated Hospital of Fujian Medical University HuiXue Zeng

Zhangzhou Affiliated Hospital of Fujian Medical University

Tiezhu Wang

Zhangzhou Affiliated Hospital of Fujian Medical University

Miaofen $\mathrm{Hu}$

Zhangzhou Affiliated Hospital of Fujian Medical University

LiJv Wang

Zhangzhou Affiliated Hospital of Fujian Medical University

\section{Zhi Wu}

Zhangzhou Affiliated Hospital of Fujian Medical University

\section{Yuming Ye}

Zhangzhou Affiliated Hospital of Fujian Medical University

\section{Yangwu Ou}

Zhangzhou Affiliated Hospital of Fujian Medical University

\section{Zhiming Cai}

Zhangzhou Affiliated Hospital of Fujian Medical University

\section{Qiyin Wu}

Zhangzhou Affiliated Hospital of Fujian Medical University

\section{Qiaozhen Xu}

Zhangzhou Affiliated Hospital of Fujian Medical University

\section{Weiliang Zhang}

Zhangzhou Affiliated Hospital of Fujian Medical University

\section{Wensen Huang}

Zhangzhou Affiliated Hospital of Fujian Medical University 


\section{Hao Li}

Zhangzhou Affiliated Hospital of Fujian Medical University

\section{Li Lin (D88385785@qq.com)}

Zhangzhou Affiliated Hospital of Fujian Medical University

\section{Research Article}

Keywords: Rapid on-site evaluation, Ultrasound bronchoscopy, Diagnostic yield, Lung biopsy, Peripheral pulmonary lesions

Posted Date: December 29th, 2021

DOI: https://doi.org/10.21203/rs.3.rs-1196540/v1

License: (c) (i) This work is licensed under a Creative Commons Attribution 4.0 International License. Read Full License 


\section{Abstract \\ Background}

To investigate the value of endobronchial ultrasound (EBUS) and virtual bronchoscopic navigation (VBN) combined with rapid on-site evaluation (ROSE) in diagnosing peripheral pulmonary lesions (PPLs).

\section{Methods}

Between January 1st 2019 to September 1st 2021, EBUS and VBN examination were performed in 250 consecutive patients with PPLs who were admitted to Zhangzhou Affiliated Hospital of Fujian Medical University (Fujian, China). Finally, 198 eligible patients were randomly divided into ROSE group (100 cases) and non-ROSE group (98 cases). The diagnostic yield of brushing and biopsy, the complications, the procedure time, the diagnosis time and expense during diagnosis were analyzed.

\section{Results}

In the ROSE group, the positive rate of EBUS brushing and biopsy were $68 \%, 84 \%$, repectively. The average procedure time and diagnosis time were $18.6 \pm 6.8 \mathrm{~min}, 3.84 \pm 4.28$ days, repectively, and the average expense was $4093.15 \pm 4494.67$ yuan. In the controls, the positive rate of brushing and biopsy were $44 \%$, $74 \%$, repectively. The average procedure time and diagnosis time were $15.4 \pm 5.7 \mathrm{~min}, 6.46 \pm 3.66$ days, repectively. And the average expense during diagnosis was $6420.28 \pm 4541.33$ yuan. There was significant difference in the positive rate of EBUS brushing and biopsy, diagnosis time and expense during diagnosis between both groups. And no significant difference was observed in the complications and the procedure time. Additionally, the impact of ROSE on diagnostic yield in right upper lobe and the size of lesion $\leq 2 \mathrm{~cm}$ in diameter was significant.

\section{Conclusion}

In combination with ROSE, EBUS could significantly improve the positive rate of diagnosing PPLs, shorten diagnosis time and reduce expense during diagnosis. ROSE will be of great importance in the diagnosis of PPLs and medical resource.

\section{Background}

Peripheral pulmonary lesions (PPLs) refer to lesions that are located in the subsegmental bronchi and cannot be visualized directly by bronchoscopy [1]. With the widespread application of computer tomography (CT), PPLs have been increasingly detected [2]. It is becoming increasingly apparent that transbronchial biopsy (TBB) has become an important method for obtaining specimen from PPLs, however, the diagnostic yield widely ranges from 36 to $76 \%$ [2-5]. The diagnosis of PPLs is still difficult 
because of its anatomic location far from segmental bronchus, which is unable to reach the lesion by routine bronchoscopy [3]. Particularly, pathological diagnosis was clinically important for those patients with benign PPLs including tuberculosis and pulmonary fungal diseases that can be distinguished from malignant lesions, which could avoid unnecessary operations and reduce medical expenses. Indeed, lung cancer as the most common PPLs is the leading cause of cancer-related death worldwide [4]. In recent years, radial endobronchial ultrasound (R-EBUS) emerged as a powerful tool during TBB and brushing in PPLs, and it led to the improvement of the diagnostic yield [5].

On the other hand, rapid on-site evaluation (ROSE) introduced by Park could help to quickly evaluate the satisfied specimen, form a preliminary diagnosis and guide the TBB operation in real time [6]. An emerging body of evidence indicates that the use of ROSE during endobronchial ultrasound-guided transbronchial needle aspiration (EBUS-TBNA) is a minimally invasive and highly accurate modality for the diagnosis of lymph node metastasis in lung cancer [7, 8]. Based on ROSE, EBUS-TBNA significantly reduced the number of needle passes and complication rates [9], which can contribute to cost savings in the medical system[10]. Actually, a high concordance rate was reported between ROSE and histologic diagnosis[11].

However, little was known about whether the utility of ROSE can affect the diagnostic yield of EBUS TBB in PPLs [12-14]. Subject to the relatively small size of sample [12] and the cohort design [13, 14], so the inference should be interpreted with coution. Additionally, the current data regarding whether several factors such as location and size of lesion[15, 16], inadequate specimen collection affecting the diagnostic yields of EBUS TBB in PPLs[17] remain confirmed.

The aim of this study was to evaluate the value of R-EBUS in combination with ROSE in diagnosing PPLs and explore factors that can influence the diagnostic yields.

\section{Methods}

\section{Participants}

This was a prospective randomized, controlled trial (RCT). Between January 1 st 2019 to September 1st 2021, EBUS and virtual bronchoscopic navigation (VBN) examination were performed in 250 consecutive patients with PPLs who were admitted to Zhangzhou Affiliated Hospital of Fujian Medical University (Fujian, China). Finally, 198 eligible patients were randomly divided into ROSE group (100 cases) and nonROSE group (98 cases). PPLs were defined as lesions surrounded by pulmonary parenchyma and endoscopically invisible (no evidence of endobronchial lesion, extrinsic compression, submucosal tumor, narrowing, inflammation, or bleeding of the bronchus) [18]. Inclusion criteria: chest CT revealed PPLs which was less than or equal to $3 \mathrm{~cm}$ in diameter. Exclusion criteria: severe emphysema, multiple or single bullae in lung parenchyma near to pulmonary lesions, cardiac or pulmonary function insufficiency, hemorrhagic diseases or coagulation disorders, and mental disorder, pregnant women, unable to stop anti-platelet aggregation drugs, patients with advanced peripheral lung cancer and other conditions that cannot cooperate with bronchoscopy. 
The study was approved by the ethical committee of Zhangzhou Affiliated Hospital of Fujian Medical University (ethics approval no. Zzsyy-2017-1116), and all patients provided informed written consent.

\section{Biopsy procedure by EBUS and VBN}

The location of the bronchus leading to the lesion was designed by VBN in advance (Ziostation2;Ziosoft Ltd, Tokyo, Japan; LungPoint; Bronchus Ltd, Mountain View, CA, USA; or DirectPath, Olympus Ltd, Tokyo, Japan). Bronchoscopy was performed applying a fiberoptic bronchoscope in combination with the REBUS (20MHz mechanical-radial type, UM-S20-20R or UM-S20-17S; Olympus, Japan) and guide sheath (GS) kit (K-201 or K-203; Olympus, Japan). The scope was inserted through the oral route, and each procedure was performed under local anesthesia with intravenous administration of midazolam for mild sedation. X-ray fluoroscopy (VersiFlex VISTA, Hitachi, Japan) was applied to guide the insertion of the REBUS probe with GS through the working channel of the bronchoscope until the target site was reached.

After determining the location of the R-EBUS probe and GS within a target lesion, brushing and TBB cytology were perfromed for specimen collection. When the R-EBUS probe was adjacent to or outside the target lesion, the bronchus closest to the PPLs was meticulously searched under fluoroscopy prior to collecting specimen. X-ray fluoroscopy guidance was applied during biopsy and brushing sampling, as well as during removal of the GS after sampling. The procedure time was measured based on the interval between insertion and removal of the bronchoscope through the vocal cords. The diagnosis time was measured based on the interval between the R-EBUS operation to final pathological diagnosis. And the expense during diagnosis indicated the costs between the R-EBUS operation to final pathological diagnosis.

\section{Rapid on-site specimen evaluation}

The material obtained from bronchoscopic biopsy or brushing, was immediately expressed onto numbered glass slides. Diff stain was applied for specimen staining (Diff-Quik; Sysmex Ltd. Kobe, Japan). The stained slide was screened by the same experienced cytopathologist, who continuously reported the findings in real time and announced when sufficient diagnostic material had been obtained for a provisional diagnosis. The bronchoscopist modified or terminated the sampling process according to the information provided by the cytopathologist. Then tissue biopsy samples were placed in $10 \%$ formalin and were embedded in paraffin for routine histologic evaluation on hematoxylin and eosin staining.

Positive diagnostic criteria for ROSE: (1) The ROSE cytology showed cancer cells and nuclear heterogeneous cells; (2) The ROSE reported neutrophils, and lesions absorpted after anti-infective treatment; (3) The ROSE showed lymphocytes and other inflammatory cells.

For those non-diagnostic patients, the final diagnosis was determined through additional medical examinations such as CT-guided percutaneous lung biopsy, surgical biopsy or anti-infection, antituberculosis therapy and follow-up for at least 6 months. 


\section{Evalutaion of complication:}

Complications are considered as follows: severe bleeding (bleeding volume $>50 \mathrm{ml})$, pneumothorax, malignant arrhythmia, lidocaine poisoning, monitoring blood oxygen saturation $<90 \%$, blood pressure greater than $180 / 120 \mathrm{mmHg}$ or less than $90 / 60 \mathrm{mmHg}$, consciousness disorder or other adverse events.

\section{Statistical analysis}

SPSS version 20.0 (SPSS, Inc., Armonk, NY, USA) was applied for statistical analyses. All variables were evaluated for normal distribution prior to analysis. Non-normally distributed continuous variables were expressed as the median (Md) and interquartile range (IQR), using Kruskal-Wallis $\mathrm{H}(\mathrm{K})$ for multiplegroup comparison. Normally distributed data were expressed as mean $\pm S D$, using a Student's $t$ test or oneway ANOVA for comparison. Categorical variables were presented as number (percentage), using the chisquare test or Fisher's exact test when compared. Differences were considered to indicate significance if a $p$ value was $<0.05$.

\section{Results}

\section{Clinical characteristics of patients}

Table 1 shows that there are 198 subjects enrolled in this study, including 116 male patients and 82 female patients; the average diameter of the lesions in the ROSE group is $2.84 \pm 2.28 \mathrm{~cm}$, and the control group is $2.48 \pm 2.66 \mathrm{~cm}$. There were 87 cases of lung tumors and 7 cases of pulmonary tuberculosis in the ROSE group, 83 cases of lung tumors and 8 cases of pulmonary tuberculosis in the non-ROSE group. No significant difference in lesion size, location of lesion and composition of disease was observed between both groups. 
Table 1

analysis of clinical characteristics in the study

\begin{tabular}{|c|c|c|c|}
\hline Characteristics & ROSE Group & Non-ROSE Group & $p$ value \\
\hline n. & 100 & 98 & \\
\hline Age (yr) & $45.23 \pm 10.34$ & $46.17 \pm 12.24$ & 0.675 \\
\hline Sex (male/female) & $59 / 40$ & $57 / 42$ & 0.847 \\
\hline Diameter (cm) & $2.84 \pm 2.28$ & $2.48 \pm 2.66$ & 0.801 \\
\hline \multicolumn{4}{|l|}{ Location of lesion } \\
\hline Right upper lobe & $18(18 \%)$ & $21(21.4 \%)$ & 0.623 \\
\hline Right middle lobe & $16(16 \%)$ & $15(15.3 \%)$ & 0.808 \\
\hline Right lower lobe & $24(24 \%)$ & $22(22.4 \%)$ & 0.821 \\
\hline Left upper lobe & $22(22 \%)$ & 18(18.7\%) & 0.693 \\
\hline Left lower lobe & $20(20 \%)$ & $22(22.4 \%)$ & 0.721 \\
\hline \multicolumn{4}{|l|}{ Final pathological diagnosis } \\
\hline Adenocarcinoma & $75(75 \%)$ & 74(75.5\%) & 0.808 \\
\hline Squamous carcinoma & $5(5 \%)$ & $4(4.1 \%)$ & 0.723 \\
\hline NSCLC, not otherwise specified & $2(2 \%)$ & $2(2.1 \%)$ & 0.921 \\
\hline Carcinoid & $2(2 \%)$ & $2(2.1 \%)$ & 0.921 \\
\hline Metastatic malignancy & $3(3 \%)$ & $1(1.0 \%)$ & 0.621 \\
\hline \multicolumn{4}{|l|}{ Benign } \\
\hline Tuberculosis & $7(7 \%)$ & $8(8.2 \%)$ & 0.813 \\
\hline Pulmonary aspergillosis & $4(4 \%)$ & $5(5.1 \%)$ & 0.723 \\
\hline Others & $2(2 \%)$ & $2(2.1 \%)$ & 0.921 \\
\hline \multicolumn{4}{|c|}{$\begin{array}{l}\text { Normally distributed data were expressed as mean } \pm S D \text {, using a Student's } t \text { test for comparison. } \\
\text { Categorical variables were presented as number (percentage), using the chi-square test or Fisher's } \\
\text { exact test when compared. Differences were considered to indicate significance if a p value was } \\
<0.05 \text {. ROSE: rapid on-site evaluation. NSCLC: non small cell lung cancer. }\end{array}$} \\
\hline
\end{tabular}

\section{The impact of ROSE on the diagnostic yield of R-EBUS brushing and biopsy}

Table 2 indicates that the positive rate of brushing in the ROSE group is $68 \%$, and the control group is $44 \%$. The positive rate of biopsy in the ROSE group is $84 \%$, and the controls is $74 \%$. The differences in the diagnostic yield of R-EBUS brushing and biopsy between both groups were significant. 
Table 2

Differerce in the diagnostic yield of R-EBUS brushing and biopsy in PPLs between both groups

\begin{tabular}{|lcccc|}
\hline & ROSE Group & Non-ROSE Group & X $^{2}$ & P value \\
\hline Diagnostic yield of brushing & $68 / 100(68 \%)$ & $44 / 98(44.9 \%)$ & 19.05 & 0.000 \\
\hline Diagnostic yield of biopsy & $84 / 100(84 \%)$ & $75 / 98(75.5 \%)$ & 8.7 & 0.001 \\
\hline $\begin{array}{l}\text { Categorical variables were presented as number (percentage), using the chi-square test or Fisher's } \\
\text { exact test when compared. Differences were considered to indicate significance if a p value was } \\
\text { e.05. ROSE: rapid on-site evaluation. PPLs: peripheral lung lesions; R-EBUS: radial endobronchial } \\
\text { ultrasound; }\end{array}$ & & & \\
\hline
\end{tabular}

\section{The Impact Of Rose On Bronchoscopy Complications}

Table 3 shows 2 cases of severe bleeding in ROSE group, and there is no significant difference in the incidence of bronchoscopy complications between both groups.

Table 3

Differerce in the incidence of bronchoscopy complications between both groups

\begin{tabular}{|lllll|}
\hline & ROSE Group & Non-ROSE Group & X & P value \\
\hline n. & 100 & 98 & & \\
\hline Positive cases & 2 & 0 & 0.990 & 0.320 \\
\hline \begin{tabular}{l} 
Incidence \\
\hline
\end{tabular} & $2 \%$ & $0 \%$ & & \\
\hline $\begin{array}{l}\text { Categorical variables were presented as number (percentage), using the chi-square test or Fisher's } \\
\text { exact test when compared. Differences were considered to indicate significance if a p value was } \\
<0.05 \text {. ROSE: rapid on-site evaluation. }\end{array}$ & & \\
\hline
\end{tabular}

\section{The impact of ROSE on the procedure time and diagnosis time}

Table 4 shows that the average procedure time in the ROSE group is $18.6 \pm 6.8 \mathrm{~min}$, and the control group is $15.4 \pm 5.7 \mathrm{~min}$. There was no significant difference in both groups. And the average diagnosis time in the ROSE group was $3.84 \pm 4.28$ days, and the controls was $6.46 \pm 3.66$ days. Significant difference was observed in both groups. 
Table 4

Differerce in the procedure time and diagnosis time between both groups

\begin{tabular}{|lccc|}
\hline & ROSE Group & Non-ROSE Group & p value \\
\hline n. & 100 & 98 & \\
\hline Procedure time & $18.6 \pm 6.8$ & $15.4 \pm 5.7$ & 0.231 \\
\hline Diagnosis time & $3.84 \pm 4.28$ & $6.46 \pm 3.66$ & 0.001 \\
\hline $\begin{array}{l}\text { Normally distributed data were expressed as mean } \pm \text { SD, using a Student's } t \text { test for } \\
\text { comparison. Differences were considered to indicate significance if a p value was <0.05. ROSE: rapid } \\
\text { on-site evaluation. }\end{array}$ & \\
\hline
\end{tabular}

\section{The impact of ROSE on the expense during diagnosis}

Table 5 shows that the average expense during diagnosis in the ROSE group is $4093.15 \pm 4494.67$ yuan, and the control group is $6420.28 \pm 4541.33$ yuan. There was statistically significant difference.

Table 5

Differerce in the expense during diagnosis between both groups

\begin{tabular}{|llcc|}
\hline & ROSE Group & Non-ROSE Group & p value \\
\hline n. & 100 & 98 & 0.011 \\
\hline Expense & $4093.15 \pm 4494.67$ & $6420.28 \pm 4541.33$ & \\
\hline $\begin{array}{l}\text { Normally distributed data were expressed as mean } \pm S D \text {, using a Student's } t \text { test for comparison. } \\
\text { Differences were considered to indicate significance if a p value was }<0.05 . \text { ROSE: rapid on-site } \\
\text { evaluation. }\end{array}$ & & \\
\hline
\end{tabular}

6. The impact of ROSE on diagnostic yield related to location and size of the lesion between both groups

Table 6 shows that the diagnostic yield of TBB in right upper lobe in the ROSE group is significantly higher than the controls. The significant difference was also observed in the size of lesion $\leq 2 \mathrm{~cm}$ in diameter.

Table 6. Diagnostic yield related to location and size of the lesion between both groups 


\begin{tabular}{|c|c|c|c|c|c|}
\hline \multirow[t]{2}{*}{ Variables } & & \multicolumn{2}{|c|}{ Diagnostic yield(\%) } & \multirow[t]{2}{*}{$x^{2}$} & \multirow[t]{2}{*}{$\mathrm{p}$ value } \\
\hline & & ROSE Group & Non-ROSE Group & & \\
\hline \multicolumn{6}{|l|}{ Location of lesion } \\
\hline Right upper lobe & & $18 / 20(90 \%)$ & $13 / 22(59.1 \%)$ & 0.505 & 0.047 \\
\hline Right middle lobe & & 14/16(87.5\%) & 14/16(87.5\%) & 0.00 & 1.000 \\
\hline Right lower lobe & & $20 / 22(90.9 \%)$ & 14/18(77.7\%) & 0.636 & 0.425 \\
\hline Left upper lobe & & $15 / 23(65.2 \%)$ & $15 / 25(60 \%)$ & 0.221 & 0.638 \\
\hline Left lower lobe & & 15/19(78.9\%) & 13/17(76.5\%) & 0.469 & 0.493 \\
\hline \multicolumn{6}{|l|}{ Size of lesion $(\mathrm{cm})$} \\
\hline \multirow[t]{2}{*}{$\leq 2$} & Brushing & $24 / 48(50 \%)$ & $12 / 46(26.1 \%)$ & 4.117 & 0.035 \\
\hline & Total diagnostic yield & $36 / 48(75 \%)$ & $26 / 46(56.5 \%)$ & 0.983 & 0.042 \\
\hline \multirow[t]{2}{*}{$>2$} & Brushing & $44 / 52(84.6 \%)$ & $40 / 52(76.9 \%)$ & 2.782 & 0.213 \\
\hline & Total diagnostic yield & $50 / 52(96.1 \%)$ & 48/52(88.9\%) & 1.020 & 0.572 \\
\hline
\end{tabular}

Categorical variables were presented as number (percentage), using the chi-square test or Fisher's exact test when compared. Differences were considered to indicate significance if a $p$ value was $<0.05$. ROSE: rapid on-site evaluation.

\section{Discussion}

Notably, the 5-year survival rate after diagnosis of lung cancer was only $18 \%$ in the United States, so early diagnosis of malignant PPLs was also of great significance [19]. Meanwhile, it is currently believed that it may be clinically important for those patients with benign PPLs distinguished from malignant lesions. Identified as an useful approach of PPLs, it was reported that the positive rate of percutaneous lung biopsy could reach $74 \%$, unfortunately, there was a high incidence of pneumothorax [20]. On the other hand, combined with VBN, the positive rate of EBUS TBB on PPLs less than $2.0 \mathrm{~cm}$ in diameter could reach $44 \%$ [21]. Therefore, clinicians were concerned about how to increase the diagnostic yield of bronchoscopy, reduce the complications and shorten the procedure time.

Particularly, ROSE has revealled clinical importance in the diagnosis of lung tumors, lung nodules, mediastinum disease and other diseases [22]. Compared with the controls [23], ROSE could reduce the number of unnecessary punctures by $33 \%$, meanwhile, it could make $68 \%$ of patients succeed in one puncture during TBNA. Although there were increasing researches on ROSE with the widespread application of TBNA, unfortunately, reports showed the controversial results [7, 24, 25]. Nakajima and his colleagues were in favor of our findings that most patients with suspected lung cancer could be diagnosed by lung biopsy pathology in combination with ROSE, and the consistency between ROSE and 
the final pathological diagnosis was $94.3 \%$ [24]. Conversely, Griffin et al. found that ROSE did not increase the positive rate of EBUS-TBNA, and it also did not reduce the number of punctures. Besides, ROSE increased expense, labor and time waste [25]. Also, there was no significant difference in diagnostic sensitivity and accuracy between ROSE group and the controls [7]. And it is now accepted that that ROSE could not increase the diagnostic yield of TBNA or EBUS-TBNA for skilled operators [26].

To the best of our knowledge, limited data are available concerning the application of ROSE during REBUS TBB in the diagnosis of PPLs [12-14, 27]. A prospective RCT enrolling 152 patients with PPLs suggested that ROSE could improve the diagnostic yield and shorten the operation time. Meanwhile, no severe procedure related complications were observed, such as pneumothorax and hemorrhage [12]. The study was clearly in favour of our results, however, we should note that the researchers did not utilize brushing in the R-EBUS procedure, and the difference in the incidence of hemorrhage between both groups was signicant. In agreement with previous studies, we found that the number of unsatisfactory specimens in ROSE group decreased $[14,17]$, then the diagnostic yield of PPLs based on ROSE expecially malignant tumors was improved [21]. However, the study conducted by Steinfort did not include control group, so the limitation may lead to some unnecessary bias [14]. Restricted to the sample size [12] and the cohort design $[13,14]$, so the conclusions should be discussed with caution.

Our findings demonstrated that ROSE could quickly evaluate whether the samples obtained are satisfactory, form a preliminary diagnosis in real time. Based on the good consistency beween ROSE and final diagnosis, the positive result supported termination of procedure without further sampling. It is obvious that EBUS combined with ROSE can reduce the operation time [12, 27]. And the improvement of the efficiency of bronchoscopy by ROSE can reduce the adverse physiological effects to some degree [28] and incidence of second biopsy. Futhermore, ROSE could show several neutrophils or macrophages or tumor cells, then bacterial culture or detailed biological characterization would be recommended [29]. In disagreement with previous study [12], Our data reported no significant difference in the operation time. But the diagnosis time was significantly shorter in ROSE group than the controls, and the. Importantly, we firstly reported the significantly decreased expense based on ROSE in combination with EBUS biopsy. And we speculated that the reduction of expense during diagnosis would be responsible for the reduced cases including severe complications and the chance of second biopsy, which contributed to public health resources greatly [30].

The majority of data suggested that some factors may affect the diagnostic yield of PPLs, such as the size of lesions and the location of lesions and so on [31]. Particularly, we were supported that ROSE can significantly improve the diagnostic yield of the lesions in diameter $\leq 2.0 \mathrm{~cm}$ [12]. And it may be attributed to the technical focus and tracheal structure reasons [31], which highlighted the clinical importance of this method in small lesions. On the other hand, supported by Steinfort [14], we observed no association between lesion size and ROSE outcome. Indeed, it should be noted that it was a cohort study and size of lesions fluctuated greatly. Besides, our study was in accordance with previous researches that the diagnostic yield of upper lobe was relatively low [32, 33]. We hypothesized that the bending angle of the upper lobe branch is too large, and the ultrasound probe is unable to stick to the 
focus and the sampling tool cannot extend to the distant focus according to the path of the probe. Under the guidance of ROSE, the extension path of biopsy forceps was modified to reach the lesion accurately, leading to an improvement of diagnostic yield in difficult cases.

There are still some limitations in our findings. Firstly, although our study was a prospective RCT, it was a single-center design, the conclusion need to be interpreted with caution. Secondly, The sample size of our study was relatively small, so it needs to be expanded to reduce unnecessary bias in the future. Thirdly, we applied the Diff-Quik staining in our study, which is a modification of the Wright-Giemsa stain, whereas other researches used modified Shorr stain for slide preparations [34]. Different staining methods were reported to be associated with varying sensitivity [35]. We may need a optimal staining method for ROSE in future studies. Fourthly, it may be a little different in every patients' examination and treatment before diagnosis, which led to some bias in expense. In spite of this, it could still reflect every patient's cost during diagnosis to a certain degree.

\section{Conclusions}

In combination with ROSE, EBUS could increase the diagnostic yield of PPLs, shorten the diagnosis time, leading to a reduction of expense during diagnosis. ROSE would be of importance in diagnosing PPLS and medical burden.

\section{Abbreviations}

PPLs: Peripheral pulmonary lesions; CT: computer tomography; TBB: transbronchial biopsy; ROSE: rapid on-site evaluation; R-EBUS: radial endobronchial ultrasound; RCT: controlled trial; IQR:interquartile range; NSCLC: Non small cell lung cancer; TBNA: transbronchial needle aspiration;VBN: virtual bronchoscopic navigation;

\section{Declarations}

Acknowledgments: We are grateful to all staff of pathology department in the pathologic diagnosis of tissues.

Funding: This work was funded by Natural Science Foundation of Fujian Province (grant 2020J01122220), Startup Fund for scientific research of Fujian Medical University (grant 2017XQ1116), and Youth Research Fund from Fujian Provincial Health Bureau (grant 2017-1-87).

Conflicts of interest: Jia-Chao Qi has received research grants from grant 2017 XQ1116 for Startup Fund for scientific research of Fujian Medical University and grant 2017-1-87 for Youth Research Fund from Fujian Provincial Health Bureau. And Li Lin has received research grant 2020J01122220 Natural Science Foundation of Fujian Province. The authors had no relevant financial or non-financial interests to disclose. Hao Li and Li Lin are the co-corresponding authors of this work. 
Ethics approval and consent to participate: All procedures performed in the studie involving human participants were in accordance with the ethical standards of the institutional and/or national research committee and with the 1964 Helsinki declaration and its later amendments or comparable ethical standards. The study was approved by the ethical committee of Zhangzhou Affiliated Hospital of Fujian Medical University. All patients provided informed written consent before the study.

Consent for publication: The authors affirm that human research participants provided informed consent for publication.

Authors' contributions: Hao Li and Li Lin conceived the study. Jia-Chao Qi, Liping Liao, Zhi-Wei Zhao and HuiXue Zeng designed and performed the experiment; analyzed the data and contributed to the manuscript preparation. LiJv Wang performed the ROSE. Tie-Zhu Wang, Miao-Fen Hu and Zhi Wu contributed to the design of the study and analyzed the data. Yu-Ming Ye, Yang-Wu Ou, Zhi-Ming Cai, QiYin Wu contributed to the revision of the manuscript. Qiao-Zhen Xu, Wei-Liang Zhang, Wen Sen Huang conducted the experiments. All authors read and approved the final manuscript.

Availability of data and materials: The datasets used and/or analysed during the current study are available from the corresponding author on reasonable request.

Competing interests: The authors declare that they have no competing interests.

\section{Author details:}

1 Department of Respiratory and Critical Care Medicine, Zhangzhou Affiliated Hospital of Fujian Medical University; No. 59, Shengli Rd., Xiangcheng, Zhangzhou, Fujian Province, People's Republic of China, 363000 .

2. Department of Ultrasonic Medicine, Zhangzhou Affiliated Hospital of Fujian Medical University; No. 59, Shengli Rd., Xiangcheng, Zhangzhou, Fujian Province, People's Republic of China, 363000.

3. Department of Otolaryngology, Zhangzhou Affiliated Hospital of Fujian Medical University; No. 59, Shengli Rd., Xiangcheng, Zhangzhou, Fujian Province, People's Republic of China, 363000.

4. Department of Pathology, Zhangzhou Affiliated Hospital of Fujian Medical University; No. 59, Shengli Rd., Xiangcheng, Zhangzhou, Fujian Province, People's Republic of China, 363000.

\section{References}

1. Shiner RJ, Rosenman J, Katz I, Reichart N, Hershko E, Yellin A: Bronchoscopic evaluation of peripheral lung tumours. Thorax 1988, 43(11):887-9.

2. National Lung Screening Trial Research T, Aberle DR, Adams AM, Berg CD, Black WC, Clapp JD et al: Reduced lung-cancer mortality with low-dose computed tomographic screening. N Engl J Med 2011, 365(5):395-409. 
3. Gould MK, Donington J, Lynch WR, Mazzone PJ, Midthun DE, Naidich DP et al: Evaluation of individuals with pulmonary nodules: when is it lung cancer? Diagnosis and management of lung cancer, 3rd ed: American College of Chest Physicians evidence-based clinical practice guidelines. Chest 2013, 143(5 Suppl):e93S-e120S.

4. Sung H, Ferlay J, Siegel RL, Laversanne M, Soerjomataram I, Jemal A et al: Global Cancer Statistics 2020: GLOBOCAN Estimates of Incidence and Mortality Worldwide for 36 Cancers in 185 Countries. CA: a cancer journal for clinicians 2021, 71(3):209-49.

5. Oki M, Saka H, Ando M, Asano F, Kurimoto N, Morita K et al: Ultrathin Bronchoscopy with Multimodal Devices for Peripheral Pulmonary Lesions. A Randomized Trial. Am J Respir Crit Care Med 2015, 192(4):468-76.

6. Davenport RD: Rapid on-site evaluation of transbronchial aspirates. Chest 1990, 98(1):59-61.

7. Oki M, Saka H, Kitagawa C, Kogure Y, Murata N, Adachi T et al: Rapid on-site cytologic evaluation during endobronchial ultrasound-guided transbronchial needle aspiration for diagnosing lung cancer: a randomized study. Respiration; international review of thoracic diseases 2013, 85(6):486-92.

8. Yang B, Li F, Shi W, Liu H, Sun S, Zhang G et al: Endobronchial ultrasound-guided transbronchial needle biopsy for the diagnosis of intrathoracic lymph node metastases from extrathoracic malignancies: a meta-analysis and systematic review. Respirology (Carlton, Vic) 2014, 19(6):83441.

9. Trisolini R, Cancellieri A, Tinelli C, Paioli D, Scudeller L, Casadei GP et al: Rapid on-site evaluation of transbronchial aspirates in the diagnosis of hilar and mediastinal adenopathy: a randomized trial. Chest 2011, 139(2):395-401.

10. Layfield LJ, Bentz JS, Gopez EV: Immediate on-site interpretation of fine-needle aspiration smears: a cost and compensation analysis. Cancer 2001, 93(5):319-22.

11. Feller-Kopman D, Yung RC, Burroughs F, Li QK: Cytology of endobronchial ultrasound-guided transbronchial needle aspiration: a retrospective study with histology correlation. Cancer 2009, 117(6):482-90.

12. Xu C, Liu W, Wang W, Li L, Hu H, Wang J: Diagnostic value of endobronchial ultrasound combined with rapid on-site evaluation of transbronchial lung biopsy for peripheral pulmonary lesions. Diagn Cytopathol 2021, 49(6):706-10.

13. Izumo T, Matsumoto Y, Sasada S, Chavez C, Nakai T, Tsuchida T: Utility of rapid on-site cytologic evaluation during endobronchial ultrasound with a guide sheath for peripheral pulmonary lesions. Japanese journal of clinical oncology 2017, 47(3):221-25.

14. Steinfort DP, Leong TL, Laska IF, Beaty A, Tsui A, Irving LB: Diagnostic utility and accuracy of rapid on-site evaluation of bronchoscopic brushings. The European respiratory journal 2015, 45(6):165360.

15. Chavez C, Sasada S, Izumo T, Watanabe J, Katsurada M, Matsumoto Y et al: Endobronchial ultrasound with a guide sheath for small malignant pulmonary nodules: a retrospective comparison between central and peripheral locations. Journal of thoracic disease 2015, 7(4):596-602. 
16. Oki M, Saka H, Kitagawa C, Kogure Y, Kajikawa S: Endobronchial Ultrasound-Guided Transbronchial Biopsy Using Novel Thin Bronchoscope for Diagnosis of Peripheral Pulmonary Lesions. Journal of thoracic oncology: official publication of the International Association for the Study of Lung Cancer 2009, 4(10):1274-77.

17. Diacon AH, Schuurmans MM, Theron J, Louw M, Wright CA, Brundyn $\mathrm{K}$ et al: Utility of rapid on-site evaluation of transbronchial needle aspirates. Respiration; international review of thoracic diseases 2005, 72(2):182-88.

18. Yamada N, Yamazaki K, Kurimoto N, Asahina H, Kikuchi E, Shinagawa N et al: Factors related to diagnostic yield of transbronchial biopsy using endobronchial ultrasonography with a guide sheath in small peripheral pulmonary lesions. Chest 2007, 132(2):603-08.

19. Ost D, Fein A: Evaluation and management of the solitary pulmonary nodule. Am J Respir Crit Care Med 2000, 162(3 Pt 1):782-87.

20. Kuban JD, Tam AL, Huang SY, Ensor JE, Philip AS, Chen GJ et al: The Effect of Needle Gauge on the Risk of Pneumothorax and Chest Tube Placement After Percutaneous Computed Tomographic (CT)Guided Lung Biopsy. Cardiovascular and interventional radiology 2015, 38(6):1595-602.

21. Ishida T, Asano F, Yamazaki K, Shinagawa N, Oizumi S, Moriya H et al: Virtual bronchoscopic navigation combined with endobronchial ultrasound to diagnose small peripheral pulmonary lesions: a randomised trial. Thorax 2011, 66(12):1072-77.

22. Chandra H, Sindhwani G, Chandra S: Role of rapid on-site evaluation with cyto-histopathological correlation in diagnosis of lung lesion. Journal of Cytology 2014, 31(4):189-93.

23. Collins BT, Chen AC, Wang JF, Bernadt CT, Sanati S: Improved laboratory resource utilization and patient care with the use of rapid on-site evaluation for endobronchial ultrasound fine-needle aspiration biopsy. Cancer Cytopathology 2013, 121(10):544-51.

24. Nakajima T, Yasufuku K, Saegusa F, Fujiwara T, Sakairi Y, Hiroshima K et al: Rapid on-site cytologic evaluation during endobronchial ultrasound-guided transbronchial needle aspiration for nodal staging in patients with lung cancer. Annals of Thoracic Surgery 2013, 95(5):1695-99.

25. Griffin AC, Schwartz LE, Baloch ZW: Utility of on-site evaluation of endobronchial ultrasound-guided transbronchial needle aspiration specimens. Cytojournal 2011, 8(8):20.

26. van der Heijden EH, Casal RF, Trisolini R, Steinfort DP, Hwangbo B, Nakajima T et al: Guideline for the acquisition and preparation of conventional and endobronchial ultrasound-guided transbronchial needle aspiration specimens for the diagnosis and molecular testing of patients with known or suspected lung cancer. Respiration; international review of thoracic diseases 2014, 88(6):500-17.

27. Xu C, Wang W, Yuan Q, Hu H, Li L, Yang R: Rapid On-Site Evaluation During Radial Endobronchial Ultrasound-Guided Transbronchial Lung Biopsy for the Diagnosis of Peripheral Pulmonary Lesions. Technology in cancer research \& treatment 2020, 19:1533033820947482.

28. Wallbridge PD, Hannan LM, Joosten SA, Irving LB, Steinfort DP: Clinical utility of sequential venous blood gas measurement in the assessment of ventilatory status during physiological stress. Internal medicine journal 2013, 43(10):1075-80. 
29. Travis WD, Rekhtman N, Riley GJ, Geisinger KR, Asamura H, Brambilla E et al: Pathologic diagnosis of advanced lung cancer based on small biopsies and cytology: a paradigm shift. Journal of thoracic oncology: official publication of the International Association for the Study of Lung Cancer 2010, 5(4):411-4.

30. Shi JF, Liu CC, Ren JS, Parascandola M, Zheng R, Tang W et al: Economic burden of lung cancer attributable to smoking in China in 2015. Tobacco control 2020, 29(2):191-99.

31. Yoshikawa M, Sukoh N, Yamazaki K, Kanazawa K, Fukumoto S, Harada M et al: Diagnostic value of endobronchial ultrasonography with a guide sheath for peripheral pulmonary lesions without $\mathrm{X}$-ray fluoroscopy. Chest 2007, 131(6):1788-93.

32. Xu CH, Wang JW, Wang W, Yuan Q, Wang YC, Chi CZ et al: The diagnosis value of endobronchial ultrasound transbronchial lung biopsy combined with rapid on-site evaluation in peripheral lung cancer. The clinical respiratory journal 2020, 14(5):447-52.

33. Shirakawa T, Imamura F, Hamamoto J, Honda I, Fukushima K, Sugimoto M et al: Usefulness of endobronchial ultrasonography for transbronchial lung biopsies of peripheral lung lesions. Respiration; international review of thoracic diseases 2004, 71(3):260-8.

34. Uchida J, Imamura F, Takenaka A, Yoshimura M, Ueno K, Oda K et al: Improved diagnostic efficacy by rapid cytology test in fluoroscopy-guided bronchoscopy. Journal of thoracic oncology: official publication of the International Association for the Study of Lung Cancer 2006, 1(4):314-8.

35. Diacon AH, Koegelenberg CF, Schubert P, Brundyn K, Louw M, Wright CA et al: Rapid on-site evaluation of transbronchial aspirates: randomised comparison of two methods. The European respiratory journal 2010, 35(6):1216-20. 